\title{
1-(3-DIMETHYLAMINOPROPYL)-3-ETHYLCARBODIIMIDE IN THE SYNTHESIS OF GLYCYRRHIZIC ACID AMINO-ACID CONJUGATES
}

\author{
L. A. Baltina, ${ }^{\text {* }}$ L. A. Baltina, Jr., ${ }^{1}$ \\ R. M. Kondratenko, ${ }^{2}$ and S. F. Petrova ${ }^{1}$
}

Glycyrrhizic acid (GA, 1) is the main triterpene glycoside from roots of Glycyrrhiza glabra L. and G. uralensis Fisch. and a leading natural glycoside with potential as a medicine for developing new immunomodulators and antiviral agents $[1,2]$. Several conjugates of GA with amino acids and dipeptides displayed pronounced antiviral activity against human immunodeficiency (HIV) [3, 4], atypical pneumonia of SARS-CoV [5], Epstein-Barr [6], and flu A/H1N1 viruses [7]. Previously, we proposed methods for preparing conjugates of GA with alkyl and benzyl esters of L- and D-amino acids via activation of the GA carboxylic acids using $N$-hydroxybenzotriazole (HOBt) and $N, N^{\prime}$-dicyclohexylcarbodiimide (DCC) [8], $N$-hydroxyphthalimide and DCC [9], or $N$-hydroxysuccinimide (HOSu) and DCC [3]. The main drawback of these methods for preparing amino-acid conjugates of GA is their two steps (preparation of GA activated ester and subsequent reaction of it with an amine). Furthermore, the dicyclohexylurea formed if DCC is used contaminates the target conjugates. Therefore, water-soluble carbodiimides, in particular 1-ethyl-3-(3-dimethylaminopropyl)carbodiimide hydrochloride (DEC), which does not form dicyclohexylurea, are more attractive as condensing reagents.

The present communication reports the selective synthesis of amino-acid conjugates of GA with free amino acids in the glycoside carbohydrate part using DEC and L-amino-acid $t$-butyl ester hydrochlorides.

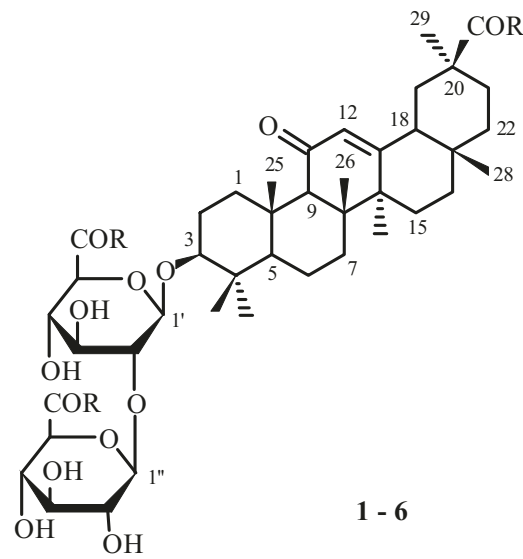

1: $\mathrm{R}=\mathrm{OH}$
2: $\mathrm{R}=\left(\mathrm{CH}_{3}\right)_{2} \mathrm{CHCH}(\mathrm{COOH}) \mathrm{NH}-(\mathrm{Val})$
3: $\mathrm{R}=\left(\mathrm{CH}_{3}\right)_{2} \mathrm{CHCH}_{2} \mathrm{CH}(\mathrm{COOH}) \mathrm{NH}-(\mathrm{Leu})$
4: $\mathrm{R}=\mathrm{CH}_{3}-\mathrm{CH}_{2} \mathrm{CH}\left(\mathrm{CH}_{3}\right) \mathrm{CH}(\mathrm{COOH}) \mathrm{NH}-(\mathrm{Ile})$
5: $\mathrm{R}=\mathrm{C}_{6} \mathrm{H}_{5}-\mathrm{CH}_{2} \mathrm{CH}(\mathrm{COOH}) \mathrm{NH}-(\mathrm{Phe})$
6: $\mathrm{R}=\mathrm{CH}_{3} \mathrm{SCH}_{2} \mathrm{CH}_{2}(\mathrm{COOH}) \mathrm{CHNH}-(\mathrm{Met})$

Condensation of GA with amino-acid $t$-butyl ester hydrochlorides (amino-component, $\mathrm{AC}$ ) was carried out at room temperature in DMF in the presence of DEC with GA-AC/DEC mole ratios 1:2.5-3.0:3.0-3.2 in the presence of an excess of $\mathrm{Et}_{3} \mathrm{~N}$. The ACs were L-amino-acid $t$-butyl esters (valine, leucine, isoleucine, phenylalanine, methionine). The obtained carboxy-substituted conjugates were deblocked by $\mathrm{CF}_{3} \mathrm{COOH}$ in $\mathrm{CH}_{2} \mathrm{Cl}_{2}$. The target GA conjugates (2-6) were isolated by column chromatography in 55-60\% yields. Compounds $\mathbf{2}$ and $\mathbf{3}$ were prepared first. The physicochemical properties of 4-6 agreed with those reported earlier [3]. The structures of 3-5 were confirmed by high-resolution PMR (500 MHz) and ${ }^{13} \mathrm{C}$ NMR spectra $(125 \mathrm{MHz}) .{ }^{13} \mathrm{C}$ NMR spectra of the obtained GA conjugates showed additional resonances for the amino-

1) Ufa Institute of Chemistry, Ufa Federal Research Center, Russian Academy of Sciences, 71 Prosp. Oktyabrya, Ufa, 450054, e-mail: baltina@anrb.ru; 2) Bashkir State Medical University, Ministry of Health of the RF, 3 Lenin St., Ufa, 450000. Translated from Khimiya Prirodnykh Soedinenii, No. 3, May-June, 2020, pp. 488-490. Original article submitted September 12, 2019. 
acid $\mathrm{COOH}$ atoms at weak field (172-175 ppm) and sets of amino-acid resonances. The resonance of the aglycon free $\mathrm{COOH}$ group appeared at $\delta 178.6-180.2 \mathrm{ppm}$. The PMR spectrum of GA conjugate 5 displayed strong multiplets for aromatic protons at weak field (7.35-7.19 ppm). The aromatic $\mathrm{C}$ atoms of phenylalanine were observed in the ${ }^{13} \mathrm{C}$ NMR spectrum at weak field (136.39-126.80 ppm). The purity of the compounds (95-99\%) was monitored by TLC and HPLC.

Thus, a simple and selective method for preparing amino-acid conjugates of GA containing free amino-acids in the glycoside carbohydrate part was proposed.

General Comments. ${ }^{13} \mathrm{C}$ NMR spectra were recorded in $\mathrm{CDCl}_{3}$ on a Bruker Avance-III pulsed spectrometer at operating frequency $500.13\left({ }^{1} \mathrm{H}\right)$ and $125.47 \mathrm{MHz}\left({ }^{13} \mathrm{C}\right)$ or a Bruker AMX-300 spectrometer at operating frequency 300 for ${ }^{1} \mathrm{H}$ and $75.5 \mathrm{MHz}$ for ${ }^{13} \mathrm{C}$. Chemical shifts were given in ppm vs. TMS internal standard. IR spectra were recorded from mineral-oil mulls on an IR Prestige-21 spectrophotometer (Shimadzu). Optical activity was measured on a PerkinElmer 341 polarimeter in a $1-\mathrm{dm}$ tube at $20-22^{\circ} \mathrm{C}\left(\lambda_{\mathrm{Na}} 546 \mathrm{~nm}\right)$.

TLC used Sorbfil plates (Sorbpolimer, Russia). Spots of compounds were detected using $\mathrm{H}_{2} \mathrm{SO}_{4}(5 \%)$ in EtOH followed by heating at $110-120^{\circ} \mathrm{C}$ for $2-3 \mathrm{~min}$. Column chromatography used KSK silica gel (50-160 $\mu \mathrm{m}$ fraction) (Sorbpolimer, Russia). HPLC used an LC-20 liquid chromatograph (Shimadzu) equipped with a spectrophotometric diode array detector at $25 \pm 2^{\circ} \mathrm{C}$ and Zorbax RX C18 reversed-phase $(250 \times 4.6 \mathrm{~mm}, 5 \mu \mathrm{m})$ (Agilent, USA) or Atlantis C1 8 columns $(250 \times 4.6 \mathrm{~mm}$, $5 \mu \mathrm{m}$ ) (Waters, USA). UV detection was made at $254 \mathrm{~nm}$. The mobile phase was $\mathrm{MeOH}$ at flow rate $1 \mathrm{~mL} / \mathrm{min}$.

GA from G. uralensis roots (Altai) was used in the work [10]. 1-Ethyl-3-(3-dimethylaminopropyl)carbodiimide hydrochloride (Sigma-Aldrich, Germany) and L-amino acid $t$-butyl esters (Chemapol, Hungary) were used in the work.

General Method for Preparing Amino-acid Conjugates of GA (2-6). A solution of $\mathbf{1}$ (0.82 g, $1 \mathrm{mmol})$ in DMF $(20 \mathrm{~mL})$ was treated with 1-ethyl-3(3-dimethylaminopropyl)carbodiimide hydrochloride (3.1-3.2 mmol), amino-acid $t$-butyl ester hydrochloride $(2.8-3.0 \mathrm{mmol})$, and $\mathrm{Et}_{3} \mathrm{~N}(0.8-1.0 \mathrm{~mL})$, stirred at $20-22^{\circ} \mathrm{C}$ for $24 \mathrm{~h}$, diluted with cold $\mathrm{H}_{2} \mathrm{O}$, and acidified with citric acid ( $\mathrm{pH} 3-4)$. The precipitate was filtered off, rinsed with $\mathrm{H}_{2} \mathrm{O}$, dried, and worked up with $\mathrm{CF}_{3} \mathrm{COOH}$ in $\mathrm{CH}_{2} \mathrm{Cl}_{2}$ $(2 \mathrm{~mL} / 10 \mathrm{~mL})$. The solvent and $\mathrm{CF}_{3} \mathrm{COOH}$ were evaporated. The solid was chromatographed over a column of silica gel with elution by $\mathrm{CHCl}_{3}-\mathrm{MeOH}-\mathrm{H}_{2} \mathrm{O}$ (400:10:1, 200:10:1, 100:10:1, 50:10:1) with TLC monitoring.

3-O-\{2-O-[N-( $\beta$-D-Glucopyranosyluronoyl)-L-valine]- $N$-( $\beta$-D-glucopyranosyluronoyl)-L-valine $\}-(3 \beta, 20 \beta)-11-$ oxo-30-norolean-12-ene (2R) (C55) (2). Yield 55\% (amorphous compound), $R_{f} 0.42\left(\mathrm{CHCl}_{3}-\mathrm{EtOH}, 5: 1\right)$; $[\alpha]_{\mathrm{D}}^{20}+55^{\circ}(c 0.06$, EtOH). IR spectrum $\left(v, \mathrm{~cm}^{-1}\right)$ : 3500-3200 (OH, NH), $1725(\mathrm{COOH}), 1660(\mathrm{C}=\mathrm{O}), 1552(\mathrm{CONH}) .{ }^{13} \mathrm{C}$ NMR spectrum (75.5 MHz, $\mathrm{CD}_{3} \mathrm{OD}, \delta$, ppm): 17.0 (C-25), 17.3 (C-24), 18.0 (C-6), 19.8 (C-26), 23.8 (C-27), 26.1 (C-2), 27.5 (C-15), 27.7 (C-16), 28.5 (C-28), 29.1 (C-23), 29.4 (C-29), 31.8 (C-21), 33.0 (C-17), 33.9 (C-7), 38.2 (C-10), 38.8 (C-22), 40.4 (C-1), 40.7 (C-4), 42.8 (C-19), 44.6 (C-14), 45.0 (C-8), 46.8 (C-20), 48.2 (C-18), 56.5 (C-5), 63.2 (C-9), 73.8, 73.7 (C-4', 4"), 75.2 (C-2"), 76.1 (C-3"), 76.4 (C-3'), 77.4 (C-5'), 77.8 (C-5"), 81.7 (C-2'), 90.3 (C-3), 104.8 (C-1'), 105.1 (C-1"'), 129.2 (C-12), 171.5, 171.6 (C-6", 6'), 172.4 (C-13), 178.6 (C-30), 202.7 (C-11); 2Val: 13.9, 18.5, 18.7, 19.5, 19.6, 31.8, 32.1, 32.2, 58.9, 58.4, 174.2 (COOH), 175.0 (COOH). Found, \%: $\mathrm{N} 2.65 . \mathrm{C}_{52} \mathrm{H}_{80} \mathrm{~N}_{2} \mathrm{O}_{18}$. Calcd, \%: N 2.74. $\mathrm{M}=1021.17$.

3-O-\{2-O-[ $N$-( $\beta$-D-Glucopyranosyluronoyl)-L-leucine]- $N$-( $\beta$-D-glucopyranosyluronoyl)-L-leucine\}-(3 $\beta, 20 \beta)-11$ oxo-30-norolean-12-ene (3). Yield 60\% (amorphous compound); $R_{f} 0.49\left(\mathrm{CHCl}_{3}-\mathrm{MeOH}-\mathrm{H}_{2} \mathrm{O}, 45: 10: 1\right)$; HPLC (95.8 $\pm 1.5 \%), \tau 2.34 \mathrm{~min}$ (Zorbax RX-C18); $[\alpha]_{\mathrm{D}}^{20}+54^{\circ}$ (c 0.04, EtOH). IR spectrum ( $\left.v, \mathrm{~cm}^{-1}\right): 3500-3200(\mathrm{OH}, \mathrm{NH}), 1727$ $(\mathrm{COOH}), 1662(\mathrm{C}=\mathrm{O}), 1540(\mathrm{CONH}) .{ }^{13} \mathrm{C}$ NMR spectrum (125 MHz, $\left.\mathrm{CD}_{3} \mathrm{OD}, \delta, \mathrm{ppm}\right): 15.6(\mathrm{C}-25), 16.0(\mathrm{C}-24), 17.1(\mathrm{C}-6)$, 18.0 (C-26), 22.6 (C-27), 26.0 (C-2), 26.2 (C-15), 26.3 (C-16), 27.0 (C-28), 27.4 (C-23), 27.9 (C-29), 30.6 (C-21), 31.6 (C-17), 32.4 (C-7), 36.7 (C-10), 37.6 (C-22), 39.0 (C-1), 39.3 (C-4), 41.0 (C-19), 43.2 (C-14), 43.5 (C-8), 45.4 (C-20), 47.4 (C-18), 55.0 (C-5), 61.7 (C-9), 71.8 (C-4"), 72.2 (C-4'), 74.3 (C-2"'), 74.6 (C-3"), 74.8 (C-3'), 75.7 (C-5'), 76.1 (C-5"), 80.5 (C-2'), 89.0 (C-3), 103.7 (C-1'), 103.8 (C-1"), 127.5 (C-12), 169.6, 170.2 (C-6", 6'), 171.6 (C-13), 179.0 (C-30), 201.1 (C-11); 2Leu: 20.7, 20.8, 22.1, 22.2, 24.5, 24.6, 40.4, 40.5, 51.2, 51.7, $174.4(\mathrm{COOH}), 174.5$ (COOH). Found, \%: N 2.54. $\mathrm{C}_{54} \mathrm{H}_{84} \mathrm{~N}_{2} \mathrm{O}_{18}$. Calcd, \%: N 2.67. $\mathrm{M}=1049.2$.

3-O-\{2-O-[N-( $\beta$-D-Glucopyranosyluronoyl)-L-isoleucine]- $N$-( $\beta$-D-glucopyranosylyronoyl)-L-isoleucine $\}$ (3 $\beta, 20 \beta)$-11-oxo-30-norolean-12-ene (4). Yield 57\% (amorphous compound), $R_{f} 0.42\left(\mathrm{CHCl}_{3}-\mathrm{EtOH}, 5: 1\right)$, HPLC $(98.8 \pm 1.5 \%), \tau 3.35 \mathrm{~min}$ (Atlantis $\mathrm{C} 18) ;[\alpha]_{\mathrm{D}}^{20}+58^{\circ}(c 0.06, \mathrm{MeOH})$. Lit. [3]: $[\alpha]_{\mathrm{D}}^{20}+60^{\circ} \mathrm{C}(c 0.04, \mathrm{MeOH})$. IR spectrum $\left(v, \mathrm{~cm}^{-1}\right): 3500-3200(\mathrm{OH}, \mathrm{NH}), 1730(\mathrm{COOH}), 1662(\mathrm{C}=\mathrm{O}), 1539(\mathrm{CONH})$. The ${ }^{13} \mathrm{C}$ NMR spectrum $\left(125 \mathrm{MHz}, \mathrm{CD}_{3} \mathrm{OD}\right.$, $\delta, \mathrm{ppm}$ ) agreed with that published [3]. Found, \%: N 2.54. $\mathrm{C}_{54} \mathrm{H}_{84} \mathrm{~N}_{2} \mathrm{O}_{18}$. Calcd, \%: $2.67 . \mathrm{M}=1049.2$.

3-O-\{2-O-[ $N$-( $\beta$-D-Glucopyranosyluronoyl)-L-phenylalanine]- $N$-( $\beta$-D-glucopyranosylyronoyl)-Lphenylalanine\}-(3 $\beta, 20 \beta$ )-11-oxo-30-norolean-12-ene (5). Yield 60\% (amorphous compound), $R_{f} 0.40\left(\mathrm{CHCl}_{3}-\mathrm{EtOH}, 5: 1\right)$; 
$[\alpha]_{\mathrm{D}}^{20}+59^{\circ}\left(c\right.$ 0.04, MeOH). Lit. [3]: $[\alpha]_{\mathrm{D}}^{20}+60^{\circ}\left(c\right.$ 0.02, MeOH). The ${ }^{13} \mathrm{C}$ NMR spectrum $\left(125 \mathrm{MHz}, \mathrm{CD}_{3} \mathrm{OD}, \delta, \mathrm{ppm}\right)$ agreed with that published [3]. Found, \%: $\mathrm{N} 2.40 . \mathrm{C}_{60} \mathrm{H}_{80} \mathrm{~N}_{2} \mathrm{O}_{18}$. Calcd, \%: N 2.50. $\mathrm{M}=1117.24$.

3-O-\{2-O-[N-( $\beta$-D-Glucopyranosyluronoyl)-L-methionine]- $N$-( $\beta$-D-glucopyranosylyronoyl)-L-methionine $\}$ (3 $\beta, 20 \beta)$-11-oxo-30-norolean-12-ene (6). Yield 56\% (amorphous compound), $R_{f} 0.42\left(\mathrm{CHCl}_{3}-\mathrm{EtOH}, 5: 1\right)$, HPLC $(99.4 \pm 1.5 \%): \tau 2.16 \min \left(Z o r b a x\right.$ RX C18); $[\alpha]_{\mathrm{D}}^{20}+54^{\circ}(c 0.05, \mathrm{MeOH})$. Lit. [3]: $[\alpha]_{\mathrm{D}}^{20}+56^{\circ}(c 0.05, \mathrm{MeOH})$. IR spectrum $\left(v, \mathrm{~cm}^{-1}\right)$ : 3450-3200 (OH, NH), $1734(\mathrm{COOH}), 1659(\mathrm{C}=\mathrm{O}), 1558(\mathrm{CONH})$. The ${ }^{13} \mathrm{C}$ NMR spectrum $\left(125 \mathrm{MHz}, \mathrm{CD}_{3} \mathrm{OD}, \delta\right.$, ppm) agreed with that published [3]. Found, \%: N 2.48, S 5.70. $\mathrm{C}_{52} \mathrm{H}_{80} \mathrm{~N}_{2} \mathrm{O}_{18} \mathrm{~S}_{2}$. Calcd, \%: N 2.58, S 5.92. $\mathrm{M}=1083.27$.

\section{ACKNOWLEDGMENT}

The work was financially supported by the RFBR (Grant 18-53-52004_MNT_a) and State Task Topic No. AAAA-A17-117011910025-6 using equipment at the Khimiya CUC, UfIC, UFRC and Agidel2 RCUC, UFRC, RAS.

\section{REFERENCES}

1. $\quad$ R. Pompei, S. Laconi, and A. Ingianni, Mini-Rev. Med. Chem., 9, 996 (2009).

2. L. A. Baltina, R. M. Kondratenko, L. A. Baltina Jr., O. A. Plyasunova, A. G. Pokrovskii, and G. A. Tolstikov, Pharm. Chem. J., 43 (10), 539 (2009).

3. L. A. Baltina Jr., R. M. Kondratenko, L. A. Baltina, N. Z. Baschenko, and O. A. Plyasunova, Russ. J. Bioorg. Chem., 35, 510 (2009).

4. L. A. Baltina Jr., E. S. Chistoedova, L. A. Baltina, R. M. Kondratenko, and O. A. Plyasunova, Chem. Nat. Compd., 48, 262 (2012).

5. G. Hoever, L. Baltina, M. Michaelis, R. Kondratenko, L. Baltina, G. A. Tolstikov, et al., J. Med. Chem., 48, 1256 (2005).

6. J.-C. Lin, J.-M. Cherng, M.-S. Hung, L. A. Baltina, L. Baltina, and R. Kondratenko, Antiviral Res., 79, 6 (2008).

7. L. A. Baltina, V. V. Zarubaev, L. A. Baltina, I. A. Orshanskaya, A. I. Fairushina, O. I. Kiselev, and M. S. Yunusov, Bioorg. Med. Chem. Lett., 25, 1742 (2015).

8. L. A. Baltina, S. A. Ryzhova, E. V. Vasil'eva, A. P. Kapina, and G. A. Tolstikov, Zh. Obshch. Khim., 63, 2140 (1993).

9. $\quad$ L. A. Baltina, A. I. Fairushina, and L. A. Baltina, Russ. J. Gen. Chem., 85, 2735 (2015).

10. O. V. Stolyarova, L. A. Baltina, Jr., L. R. Mikhlailova, T. M. Gabbasov, L. A. Baltina, and G. A. Tolstikov, Chem. Sustainable Develop., 16, 563 (2008). 